\title{
Dielectric and electrical characteristics of mechanically synthesized Ni-Zn ferrite nanoparticles
}

\begin{abstract}
$\mathrm{Ni}-\mathrm{Zn}$ ferrite nanoparticles were synthesized via mechanical activation of $\mathrm{Zn}, \mathrm{NiO}$ and $\mathrm{Fe} 2 \mathrm{O} 3$ powders in a high energy planetary ball mill. The $30 \mathrm{~h}$-milled samples in argon, oxygen and air atmospheres were pressed in pellet and toroid shape form and were sintered from $500{ }^{\circ} \mathrm{C}$ to $900{ }^{\circ} \mathrm{C}$ with $100{ }^{\circ} \mathrm{C}$ increments. The X-ray diffraction patterns results indicated a single phase $\mathrm{Ni}-\mathrm{Zn}$ ferrite formation with a cubic-spinel structure in all the samples sintered at $500{ }^{\circ} \mathrm{C}$. The milling atmosphere had a key role in the synthesis, microstructure and properties of the samples in such a way that this effect sustained even after the completion of sintering process. Thus, the main goal of this study is to scrutinize the effect of sintering temperature in the 30-h-milled samples in different atmospheres on DC electrical resistivity and dielectric behavior of Ni-Zn ferrite samples. The results indicated that although electrical resistivity decreased, dielectric behaviors, i.e. constant, loss and tan increased with increase in sintering temperature. The milled samples in argon had the highest resistivity of $1.2106 \Omega \mathrm{cm}$ at $500{ }^{\circ} \mathrm{C}$, and lowest dielectric constant and loss (4.67 102 and 1.7 at $300 \mathrm{~K}$ and frequency $106 \mathrm{MHz}$, respectively) compared to other samples owing to more homogeneity and smaller average crystallite size, making them a good candidate for high frequency applications. X-ray photoelectron spectroscopy (XPS) revealed the presence of metal ions in their proper valence in the Ni-Zn ferrite crystal structure. Noticeably, a variation in the binding energy for the milled samples in different atmospheres is attributed to the changes in surroundings of $\mathrm{Fe} 3+$ and $\mathrm{Zn} 2+/$ or $\mathrm{Ni} 2+$, due to non-equilibrium distribution of cations in tetrahedral and octahedral sites, which is further confirmed by the XRD patterns.
\end{abstract}

Keyword: Ni-Zn ferrite; Electrical properties; Milling atmosphere; Sintering; Dielectric properties; XPS analysis 